Bull. Korean Math. Soc. 50 (2013), No. 3, pp. 747-752

http://dx.doi.org/10.4134/BKMS.2013.50.3.747

\title{
ON DISCRETENESS OF MÖBIUS GROUPS
}

\author{
XI FU
}

ABStRaCt. It's known that one could use a fixed loxodromic or parabolic element in $M\left(\overline{\mathbb{R}}^{n}\right)$ as a test map to test the discreteness of a nonelementary Möbius group $G$. In this paper, we discuss the discreteness of $G$ by using a fixed elliptic element.

\section{Introduction}

The discreteness of Möbius groups is an old and interesting problem which has been studied by many authors. For instance, in [4], Jørgensen obtained a useful necessary condition for two-generator Möbius groups of $\operatorname{PSL}(2, \mathbb{C})$, which is known as Jørgensen's inequality. As an application, he established the following discreteness criterion in [5].

Theorem J. A non-elementary subgroup $G$ of $P S L(2, \mathbb{C})$ is discrete if and only if every two-generator subgroup in $G$ is discrete.

This implies that the discreteness of a non-elementary Möbius group $G$ depends on the discreteness of its two-generator subgroups. There are many further discussions in this direction (see [6], [8], [11]). In 2001, Wang and Yang [12] generalized Theorem $\mathrm{J}$ to the case of $M\left(\overline{\mathbb{R}}^{n}\right)$ and proved the following.

Theorem WY. Let $G \subset M\left(\overline{\mathbb{R}}^{n}\right)$ be non-elementary. Then $G$ is discrete if and only if $W Y(G)$ is discrete and each non-elementary subgroup generated by two loxodromic elements in $G$ is discrete.

Here,

$W Y(G)=\{g \in G: g$ fixes every fixed point of each loxodromic element of $G\}$.

Obviously, if $G \subset P S L(2, \mathbb{C})$ is non-elementary, then $W Y(G)=\{I\}$. According to [12], we know that the condition "WY $G)$ is discrete" in Theorem WY is necessary when $n \geq 3$.

Received February 13, 2012.

2010 Mathematics Subject Classification. Primary 30F40; Secondary 20H10, 57S30.

Key words and phrases. discreteness, elliptic elements, loxodromic elements.

The research was partially supported by Tian-Yuan Foundation (No. 11226096) and NSF of China (No. 11071063). 
In [10], Wang, Li and Cao obtained further generalizations of Theorem WY. By using a fixed loxodromic or parabolic element in $G$, they proved the following theorems.

Theorem $\mathbf{W}_{1}$ ([10, Theorem 3.1]). Let $G \subset M\left(\overline{\mathbb{R}}^{n}\right)$ be non-elementary. Then $G$ is discrete if and only if $W Y(G)$ is discrete and each non-elementary subgroup generated by two elements of $G_{f}$ is discrete, where $f \in G$ is loxodromic.

Theorem $\mathbf{W}_{2}\left(\left[10\right.\right.$, Theorem 3.2]). Let $G \subset M\left(\overline{\mathbb{R}}^{n}\right)$ be non-elementary containing parabolic elements. Then $G$ is discrete if and only if $W Y(G)$ is discrete and each non-elementary subgroup generated by two elements of $G_{f}$ is discrete, where $f \in G$ is parabolic.

Here $G_{f}$ in Theorems $W_{1}$ and $W_{2}$ are defined as follows:

$G_{f}=\{g \in G: g$ is conjugate to $f$ and $\langle f, g\rangle$ is non-elementary $\} \cup\{f\}$.

The novelty of Theorems $W_{1}$ and $W_{2}$ is that the discreteness of $G$ is totally determined by a loxodromic (resp. parabolic) element of $G$.

In [7], Li and the author showed that the assumption " $f \in G$ " in Theorems $W_{1}$ and $W_{2}$ was unnecessary and obtained the following theorems.

Theorem $\mathbf{L F}_{\mathbf{1}}$ ([7, Theorem 1.1]). Let $G \subset M\left(\overline{\mathbb{R}}^{n}\right)$ be a non-elementary group and $f \in M\left(\overline{\mathbb{R}}^{n}\right)$ loxodromic. If $W Y(G)$ is discrete and each non-elementary group $\left\langle f, g f g^{-1}\right\rangle$ is discrete, where $g \in G$, then $G$ is discrete.

Theorem $\mathbf{L F}_{\mathbf{2}}\left(\left[7\right.\right.$, Theorem 1.2]). Let $G \subset M\left(\overline{\mathbb{R}}^{n}\right)$ be a non-elementary group and $f \in M\left(\overline{\mathbb{R}}^{n}\right)$ parabolic. If $W Y(G)$ is discrete and each non-elementary group $\left\langle f, g f g^{-1}\right\rangle$ is discrete, where $g \in G$, then $G$ is discrete.

Naturally, we asked the following question.

Conjecture LF $([7])$. Let $G \subset M\left(\overline{\mathbb{R}}^{n}\right)$ be a non-elementary group and $f \in$ $M\left(\overline{\mathbb{R}}^{n}\right)$ elliptic. If $W Y(G)$ is discrete, and each non-elementary group

$$
\left\langle f, g f g^{-1}\right\rangle
$$

is discrete, where $g \in G$, then $G$ is discrete.

We constructed an example in [7] which showed that if $\left.f\right|_{M(G)}=I$, then Conjecture LF may not be true.

Example LF ([7]). Let $G_{0} \subset M\left(\overline{\mathbb{R}}^{2}\right)$ be a non-elementary and non-discrete group containing no elliptic elements, and let $G$ be the Poincaré extension of $G_{0}$ in $\overline{\mathbb{R}}^{4}$. Let $f$ be a rotation around $\overline{\mathbb{R}}^{2}$ of order $p(p \geq 3)$. Then $f$ is an elliptic element acting on $\overline{\mathbb{R}}^{4}$ whose fixed point set is $\overline{\mathbb{R}}^{2}$. Obviously, $f \notin G$, $W Y(G)=\langle I\rangle$ is finite but there exists no non-elementary group generated by $f$ and $g f g^{-1}$ for $g \in G$.

In this paper, we discuss Conjecture LF further and some new discreteness criteria of Möbius groups are obtained. 


\section{Preliminaries}

For $n \geq 2$, we denote by $\overline{\mathbb{R}}^{n}$ the one-point compactification of $\mathbb{R}^{n}$ obtained by adding $\infty$. The group of orientation-preserving Möbius transformations of $\overline{\mathbb{R}}^{n}$ is denoted by $M\left(\overline{\mathbb{R}}^{n}\right)$. We regard $\overline{\mathbb{R}}^{n}$ as the boundary at infinity of the hyperbolic $(n+1)$-space $\mathbb{H}^{n+1}$ and let $\overline{\mathbb{H}}^{n+1}=\mathbb{H}^{n+1} \cup \overline{\mathbb{R}}^{n}$. It's known that every Möbius transformation $f$ in $\overline{\mathbb{R}}^{n}$ can be extended to an isometry $\widetilde{f}$ (Poincaré extension) in $\mathbb{H}^{n+1}$.

For a non-trivial element $f \in M\left(\overline{\mathbb{R}}^{n}\right)$, we let

$$
f i x(f)=\left\{x \in \overline{\mathbb{H}}^{n+1}: f(x)=x\right\}
$$

$f$ is called loxodromic if it has two fixed points in $\overline{\mathbb{R}}^{n}$ and none in $\mathbb{H}^{n+1}$, parabolic if it has only one fixed point in $\overline{\mathbb{R}}^{n}$ and none in $\mathbb{H}^{n+1}$, and elliptic if it has a fixed point in $\mathbb{H}^{n+1}$.

Let $G$ be a subgroup of $M\left(\overline{\mathbb{R}}^{n}\right)$. For a point $z \in \overline{\mathbb{H}}^{n+1}$, the set $G(z)=$ $\{g(z): g \in G\}$ is called $G$-orbit of $z$. The limit set $L(G)$ of $G$ is defined as follows:

$$
L(G)=\overline{G(z)} \cap \overline{\mathbb{R}}^{n} .
$$

We call $G$ elementary if $L(G)$ contains fewer than three points. Otherwise, it is called non-elementary.

Proposition 2.1 ([10]). Let $G \subset M\left(\overline{\mathbb{R}}^{n}\right)$. Then we have the following

(1) if $G$ contains a loxodromic element, then $G$ is elementary if and only if it fixes a point in $\overline{\mathbb{R}}^{n}$ or a point-pair $\{x, y\} \subset \overline{\mathbb{R}}^{n}$;

(2) if $G$ contains a parabolic element but no loxodromic element, then $G$ is elementary if and only if it fixes a point in $\overline{\mathbb{R}}^{n}$;

(3) if $G$ is purely elliptic, then $G$ fixes a point in $\overline{\mathbb{H}}^{n+1}$.

Let $G \subset M\left(\overline{\mathbb{R}}^{n}\right)$ be non-elementary. We denote $M(G)$ the smallest $G$ invariant hyperbolic subspace of $\mathbb{H}^{n+1}, \phi(g)$ the restriction of $g$ to $M(G)$ for all $g \in G$, that is

$$
\phi(g)=\left.g\right|_{M(G)}, \phi(G)=\left\{\left.g\right|_{M(G)}: g \in G\right\} .
$$

Obviously,

$$
W Y(G)=\{g \in G: \phi(g)=I\} .
$$

If there exists a sequence of distinct elements in $G$ converging to the identity, then we say that $G$ is not discrete. Otherwise, we say that $G$ is discrete.

Proposition $2.2([9])$. Let $G \subset M\left(\overline{\mathbb{R}}^{n}\right)$ be non-elementary. Then $G$ is discrete if and only if both groups $W Y(G)$ and $\phi(G)$ are discrete.

For $f_{r}=\left(\begin{array}{ll}a_{r} & b_{r} \\ c_{r} & d_{r}\end{array}\right) \in M\left(\overline{\mathbb{R}}^{n}\right)(r=1,2)$, we define

$$
\left\|f_{1}-f_{2}\right\|=\left(\left|a_{1}-a_{2}\right|^{2}+\left|b_{1}-b_{2}\right|^{2}+\left|c_{1}-c_{2}\right|^{2}+\left|d_{1}-d_{2}\right|^{2}\right)^{\frac{1}{2}} .
$$

The following lemma is crucial for our investigation. 
Lemma 2.1 ([13]). Let $f, g \in M\left(\overline{\mathbb{R}}^{n}\right)$. If $\langle f, g\rangle$ is a discrete and nonelementary group, then

$$
\|f-I\| \cdot\|g-I\|>\frac{1}{32}
$$

In the following, we give an example which shows that in some special case, Conjecture LF may be true.

Proposition 2.3. Let $G \subset M\left(\overline{\mathbb{R}}^{5}\right)$ be non-elementary with $M(G)=\mathbb{H}^{6}$, and let $f$ be an elliptic element of $M\left(\overline{\mathbb{R}}^{5}\right)$ such that $f^{2}$ has only one fixed point in $\mathbb{H}^{6}$ and none in $\overline{\mathbb{R}}^{5}$. If each non-elementary group $\left\langle f, g f g^{-1}\right\rangle$ is discrete, where $g \in G$, then $G$ is discrete.

Proof. Suppose not. Since $G \subset M\left(\overline{\mathbb{R}}^{5}\right)$ is non-elementary with $M(G)=\mathbb{H}^{6}$, by [3], we know that $G$ is dense in $M\left(\overline{\mathbb{R}}^{5}\right)$. It follows that there exists a sequence $\left\{f_{i}\right\} \subset G$ such that for each $i, f_{i}$ is loxodromic and

$$
f_{i} \rightarrow I \text { as } i \rightarrow \infty \text {. }
$$

By our assumptions and Lemma 2.1, it's easy to see that for large enough $i$, $\left\langle f^{2}, f_{i} f^{2} f_{i}^{-1}\right\rangle$ are elementary. It deduces that

$$
f_{i}\left(f i x\left(f^{2}\right)\right)=f i x\left(f^{2}\right) .
$$

It's the desired contradiction since $f^{2}$ has only one fixed point in $\mathbb{H}^{6}$.

Motivated by Example LF and Proposition 2.3, we obtain the following main results.

Theorem 2.1. Let $G \subset M\left(\overline{\mathbb{R}}^{n}\right)$ be non-elementary and $f \in M\left(\overline{\mathbb{R}}^{n}\right)$ be elliptic with Card $\left[\right.$ fix $\left.\left(f^{2}\right)\right]=1$. If $W Y(G)$ is discrete, and each non-elementary group $\left\langle f, g f g^{-1}\right\rangle$ is discrete, where $g \in G$, then $G$ is discrete.

Theorem 2.2. Let $G \subset M\left(\overline{\mathbb{R}}^{n}\right)$ be non-elementary and $f \in M\left(\overline{\mathbb{R}}^{n}\right)$ be elliptic with $\left.f^{2}\right|_{M(G)} \neq I$. If $W Y(G)$ is discrete, and each non-elementary group $\langle f, g\rangle$ is discrete, where $g \in G$, then $G$ is discrete.

Remark 2.1. Following [3], if $f \in M\left(\overline{\mathbb{R}}^{n}\right)$ be elliptic with $\operatorname{Card}[f i x(f)]=1$, then $n$ must be odd.

\section{Proofs of main results}

\subsection{Proof of Theorem 2.1}

Suppose not. Then there exists a sequence $\left\{f_{i}\right\} \subset G$ such that

$$
f_{i} \rightarrow I \text { as } i \rightarrow \infty \text {. }
$$

Since $\operatorname{Card}\left[f i x\left(f^{2}\right)\right]=1$, we can see that $f^{2}$ has no fixed point in $\overline{\mathbb{R}}^{n}$ (that is $\widetilde{f^{2}}$ has only one fixed point in $\left.\mathbb{H}^{n+1}\right)$. Without loss of generality, we assume 
that $f i x\left(f^{2}\right)=\{x\}$, where $x \in \mathbb{H}^{n+1}$. Then for large enough $i$, we know that the subgroups $\left\langle f^{2}, f_{i} f^{2} f_{i}^{-1}\right\rangle$ are elementary since

$$
\left\|f^{2}-I\right\| \cdot\left\|f^{-2} f_{i} f^{2} f_{i}^{-1}-I\right\|<\frac{1}{32} .
$$

By Proposition 2.1, we know that for large enough $i, x \in f i x\left(f_{i}\right)$. Since $G$ is non-elementary, we can find finitely many loxodromic elements $g_{1}, g_{2}, \ldots, g_{t}$ in $G$ such that the set $S=\left\{A_{f i x\left(g_{1}\right)}, A_{f i x\left(g_{2}\right)}, \ldots, A_{f i x\left(g_{t}\right)}\right\}$ can spans $M(G)$, where $A_{f i x(g)}$ denote the attractive fixed point of a loxodromic element $g$. For each $k$, let $U_{A_{f i x\left(g_{k}\right)}}$ be a small neighborhood of $A_{f i x\left(g_{k}\right)}$ in $\overline{\mathbb{H}}^{n+1}$, where $(k=$ $1,2, \ldots, t)\left(\right.$ cf. [7]). Then we can find an integer $N$ such that for each $k, g_{k}^{N}(x) \in$ $U_{A_{f i x\left(g_{k}\right)}}$. Now, let's consider the subgroups $\left\langle g_{t}^{N} f^{2} g_{t}^{-N}, f_{i} g_{t}^{N} f^{2} g_{t}^{-N} f_{i}^{-1}\right\rangle$. Since

$$
\left\langle g_{t}^{N} f^{2} g_{t}^{-N}, f_{i} g_{t}^{N} f^{2} g_{t}^{-N} f_{i}^{-1}\right\rangle=g_{t}^{N}\left\langle f^{2}, g_{t}^{-N} f_{i} g_{t}^{N} f^{2} g_{t}^{-N} f_{i}^{-1} g_{t}^{N}\right\rangle g_{t}^{-N}
$$

and

$$
\left\langle f^{2}, g_{t}^{-N} f_{i} g_{t}^{N} f^{2} g_{t}^{-N} f_{i}^{-1} g_{t}^{N}\right\rangle=\left\langle f^{2}, g_{t}^{-N} f_{i} g_{t}^{N} f^{2} g_{t}^{-N} f_{i}^{-1} g_{t}^{N} f^{-2}\right\rangle,
$$

by the assumptions and Lemma 2.1, we know that the groups

$$
\left\langle g_{t}^{N} f^{2} g_{t}^{-N}, f_{i} g_{t}^{N} f^{2} g_{t}^{-N} f_{i}^{-1}\right\rangle
$$

are elementary for large enough $i$. It easily follows that for each $k$ and large enough $i, f_{i}$ has a fixed point in $U_{A_{f i x\left(g_{k}\right)}}$. This means that for large enough $i$, $f_{i} \in W Y(G)$. It's a contradiction.

\subsection{Proof of Theorem 2.2}

Suppose that $G$ is not discrete. Then there exists a sequence $\left\{f_{i}\right\} \subset G$ such that for each $i$,

$$
f_{i} \rightarrow I \quad \text { as } i \rightarrow \infty .
$$

It follows a discussion similar to that in the proof of Theorem 2.1, we can find finitely many loxodromic elements $g_{1}, g_{2}, \ldots, g_{t}$ in $G$ such that the set $S=\left\{A_{f i x\left(g_{1}\right)}, A_{f i x\left(g_{2}\right)}, \ldots, A_{f i x\left(g_{t}\right)}\right\}$ can span $M(G)$ and an integer $N$ such that for each $k, g_{k}^{N}(f i x(f)) \in U_{A_{f i x\left(g_{k}\right)}}(k=1,2, \ldots, t)$. Since

$$
\left\langle g_{k}^{N} f^{2} g_{k}^{-N}, f_{i}\right\rangle=g_{k}^{N}\left\langle f^{2}, g_{k}^{-N} f_{i} g_{k}^{N}\right\rangle g_{k}^{-N}
$$

and

$$
\left\|g_{k}^{-N} f_{i} g_{k}^{N}-I\right\| \cdot\left\|f^{2}-I\right\|<\frac{1}{32}
$$

for large enough $i$, we can see that the subgroups $\left\langle g_{k}^{N} f^{2} g_{k}^{-N}, f_{i}\right\rangle$ are elementary. By Proposition 2.1, we know that for each $k(k=1,2, \ldots, t)$,

$$
f i x\left(f_{i}\right) \cap U_{A_{f i x\left(g_{k}\right)}} \neq \emptyset .
$$

It follows that for sufficiently large $i$,

$$
f_{i} \in W Y(G) .
$$


It's a contradiction.

\section{A discreteness criterion for isometric subgroups of $\mathrm{PU}(\mathbf{n}, 1)$}

It's similar to Möbius groups, in [6], Li obtained the following discreteness criteria for subgroups of $P U(n, 1)$.

Theorem $\mathbf{L}([6$, Theorem 1.3]). Let $G \subset P U(n, 1)$ be non-elementary and $M(G)=\mathbb{H}_{\mathbb{C}}^{n}$. Suppose that $f \in G$ is elliptic with order at least 3. Then $G$ is discrete if and only if each non-elementary subgroup generated by $f$ and an elliptic of $G$ is discrete.

By [3], we know that if $M(G)=\mathbb{H}_{\mathbb{C}}^{n}$, then $G$ is either discrete or dense. Since $\operatorname{dim}(M(G))$ is even, it follows from a discussion similar to that in the proof of [2, Theorem 1.2], we have:

Theorem 4.1. Let $G \subset P U(n, 1)$ be non-elementary and $M(G)=\mathbb{H}_{\mathbb{C}}^{n}$. Then $G$ is discrete if and only if each group generated by an elliptic element of $G$ is discrete.

\section{References}

[1] A. F. Beardon, The Geometry of discrete groups, Graduate text in Mathematics, Vol. 91, Springer-Verlag, 1983.

[2] W. Cao and X. Wang, Discreteness criteria and algebraic convergence theorem for subgroups in $P U(1, n ; \mathbb{C})$, Proc. Japan Acad. Ser. A Math. Sci. 82 (2006), no. 3, 49-52.

[3] S. S. Chen and L. Greenberg, Hyperbolic spaces, Contributions to analysis (a collection of papers dedicated to Lipman Bers), pp. 49-87. Academic Press, New York, 1974.

[4] T. Jørgensen, On discrete groups of Möbius transformation, Amer. J. Math. 98 (1976), no. $3,739-749$

[5] — A note on subgroups of $S L(2, \mathbb{C})$, Quart. J. Math. Oxford Ser. (2) 28 (1977), no. 110, 209-211.

[6] L. Li, Elliptic elements in Möbius groups, Proc. Japan Acad. Ser. A Math. Sci. 86 (2010), no. 3, 69-72.

[7] L. Li and X. Fu, Discreteness by use of a test map, Bull. Korean. Math. Soc. 49 (2012), no. $1,57-61$.

[8] P. Tukia and X. Wang, Discreteness of subgroups of $S L(2, \mathbb{C})$ containing elliptic elements, Math. Scand. 91 (2002), no. 2, 214-220.

[9] X. Wang, Dense subgroups of n-dimensional Möbius groups, Math. Z. 243 (2003), no. 4, 643-651.

[10] X. Wang, L. Li, and W. Cao, Discreteness criteria for Möbius groups acting on $M\left(\overline{\mathbb{R}}^{n}\right)$, Israel J. Math. 150 (2005), 357-368.

[11] X. Wang and W. Yang, Discreteness criterions for subgroups in $\mathrm{SL}(2, \mathbf{C})$, Math. Proc. Cambridge Philos. Soc. 124 (1998), no. 1, 51-55.

[12] _ Discreteness criteria of Möbius groups of high dimensions and convergence theorems of Kleinian groups, Adv. Math. 159 (2001), no. 1, 68-82.

[13] P. Waterman, Möbius transformations in several dimensions, Adv. Math. 101 (1993), no. $1,87-113$

Department of Mathematics

SHAOXING UNIVERSITY

Shaoxing, Zhejiang 312000, P. R. China

E-mail address: fuxi1000@yahoo.com.cn 